\title{
MARINE RESOURCES RESEARCH INSTITUTE RADIOCARBON DATES III*
}

\author{
THOMAS D MATHEWS
}

Marine Resources Research Institute, PO Box 12559

Charleston, South Carolina 29412

Samples in this paper are a continuation of the Florida geologic samples, reported earlier ( $R, 1978$, v 20, p 436-440). Each specimen analyzed in this list was a carbonate shell. All dates are from single shells as opposed to means of multiple shell samples.

As previously discussed ( $R, 1978, \mathrm{v} 20, \mathrm{p}$ 436-440), wide age ranges were found for the various Holocene beach deposits. Very old samples $(>20,000 \mathrm{yr}$ BP) were found in conjunction with comparatively young samples (2000 to $4000 \mathrm{yr}$ BP), presumably due to reworking and incorporation of older deposits with younger ones.

Analytic procedures and age calculations were performed as previously reported ( $R, 1976, v$ 18, p 202-204). All ages were based on a ${ }^{14} \mathrm{C}$ half-life of 5570 years, using $0.95 \mathrm{NBS}$ oxalic acid as the modern standard. Each sample was counted a minimum of 2000 minutes. Calculations were based on sample, standard, and background statistics to $\pm 1 \sigma$.

After experimenting with laboratory procedures, the best overall results were obtained by utilizing a $\mathrm{V}_{2} \mathrm{O}_{5}$-alumina catalyst, prepared basically as described by Coleman et al (1972). Rather than heating the catalyst in a muffle furnace for $48 \mathrm{hr}$ at $550^{\circ} \mathrm{C}$, it is heated in a tubefurnace at $550^{\circ} \mathrm{C}$ for 1 to $2 \mathrm{hr}$ in a stream of oxygen. Acetylene is allowed to sublime directly onto this catalyst after the catalyst has been heated in vacuo to remove adsorbed oxygen. Samples of benzene in the 2.5 to $3 \mathrm{~g}$ range can be formed from the acetylene in 30 to 45 minutes. Overall yields are generally 70 to $80 \%$, but occasionally may be $>80 \%$. This yield is somewhat low, possibly because of incomplete reaction in the $\mathrm{CO}_{2}$-lithium step, rather than because of the catalyst.

\section{ACKNOWLEDGMENTS}

Field work was conducted primarily by F W Stapor, Jr. Thanks are extended to $\mathrm{C}$ DuPree for typing the manuscript.

\section{SAMPLE DESCRIPTIONS}

\section{Siesta Key, Sarasota Co}

Samples coll from lithified calcarenite at Point-of-Rocks on Siesta Key $\left(27^{\circ} 14^{\prime} 42^{\prime \prime} \mathrm{N}, 82^{\circ} 32^{\prime} 14^{\prime \prime} \mathrm{W}\right)$ at MSL.

\section{MRRI-102. Shell (Mercenaria sp) $2520 \pm 80$ \\ MRRI-104. Shell (Mercenaria sp) $2500 \pm 110$ \\ MRRI-106. Shell (Mercenaria sp) $\quad 3140 \pm 110$}

* Contribution No. 122 from the South Carolina Marine Resources Center 
MRRI-119. Shell (Mercenaria sp)

$$
4590 \pm 210
$$

MRRI-120. Shell (Mercenaria sp)

$$
4590 \pm 90
$$

\section{Gasparilla I., Lee Co}

Samples coll from lithified calcarenite at Boca Grande on Gasparilla I. $\left(26^{\circ} 45^{\prime} 48^{\prime \prime} \mathrm{N}, 82^{\circ} 15^{\prime} 55^{\prime \prime} \mathrm{W}\right)$ at MSL.

\section{MRRI-122. Shell (Mercenaria sp)}

$$
5550 \pm 140
$$

MRRI-123. Shell (Mercenaria sp)

$$
4240 \pm 320
$$

MRRI-124. Shell (Mercenaria sp)

$6910 \pm 290$

MRRI-125. Shell (Mercenaria sp)

$$
2110 \pm 110
$$

\section{Cape Canaveral Launch Complex No. 19}

Samples coll from beach ridge with poorly lithified calcarenite 0 to $1 \mathrm{~m}$ above MLW (28 $\left.30^{\prime} 26^{\prime \prime} \mathrm{N}, 80^{\circ} 33^{\prime} 00^{\prime \prime} \mathrm{W}\right)$.

MRRI-130. Shell (Anadara brasiliana)

$$
\begin{array}{r}
19,300 \pm 970 \\
4210 \pm 80 \\
20,100 \pm 640 \\
5030 \pm 290 \\
5550 \pm 240 \\
6990 \pm 370 \\
4580 \pm 170 \\
7370 \pm 220 \\
21,100 \pm 900
\end{array}
$$$$
\text { MRRI-131. Shell (Busycon carica) }
$$$$
\text { MRRI-132. Shell (B carica) }
$$

MRRI-134. Shell (A ovalis)

MRRI-136. Shell (A brasiliana)

MRRI-138. Shell (Dinocardium robustum)

MRRI-143. Shell (Anadara sp)

MRRI-144. Shell (Anadara sp)

MRRI-145. Shell (B carica)

Launch Complex No. 11

Samples coll from unconsolidated sand 1 to $2 \mathrm{~m}$ above MSL $\left(28^{\circ}\right.$ $28^{\prime} 55^{\prime \prime} \mathrm{N}, 80^{\circ} 31^{\prime} 45^{\prime \prime} \mathrm{W}$ ).

$\begin{array}{ll}\text { MRRI-161. } & \text { Shell (B carica) } \\ \text { MRRI-162. } & \text { Shell (Anadara } \mathbf{s p )} \\ \text { MRRI-163. } & \text { Shell (Mercenaria } \mathbf{s p} \text { ) } \\ \text { MRRI-164. } & \text { Shell (B carica) } \\ \text { MRRI-165. } & \text { Shell (B carica) } \\ \text { MRRI-166. } & \text { Shell (B carica) } \\ \text { MRRI-167. } & \text { Shell (Anadara } \mathbf{s p )} \\ \text { MRRI-168. } & \text { Shell (B carica) } \\ \text { MRRI-169. } & \text { Shell (B carica) } \\ \text { MRRI-170. } & \text { Shell (B carica) }\end{array}$

$$
\begin{aligned}
20,600 & \pm 480 \\
3920 & \pm 130 \\
4900 & \pm 120 \\
19,700 & \pm 400 \\
2030 & \pm 80 \\
6110 & \pm 180 \\
20,000 & \pm 710 \\
17,200 & \pm 600 \\
5090 & \pm 130 \\
18,300 & \pm 380
\end{aligned}
$$


Sanibel I., Lee Co Location No. 1

Samples coll from beach ridge sand $\sim 70 \mathrm{~cm}$ above MLW $\left(26^{\circ} 28^{\prime}\right.$ $\left.15^{\prime \prime} \mathrm{N}, 82^{\circ} 09^{\prime} 30^{\prime \prime} \mathrm{W}\right)$.

MRRI-139. Shell (Mercenaria sp)

$4420 \pm 160$

MRRI-146. Shell (D robustum)

$5280 \pm 160$

MRRI-149. Shell (Anadara sp)

$2410 \pm 100$

MRRI-150. Shell (B carica)

$2520 \pm 120$

MRRI-153. Shell (Mercenaria sp)

$2090 \pm 80$

MRRI-157. Shell (D robustum)

$2860 \pm 90$

MRRI-159. Shell (Mercenaria sp)

$3990 \pm 290$

\section{Location No. 3}

Samples coll from beach ridge sand $\sim 70 \mathrm{~cm}$ above MLW $\left(26^{\circ} 27^{\prime}\right.$ $26^{\prime \prime} \mathrm{N}, 82^{\circ} 09^{\prime} 12^{\prime \prime} \mathrm{W}$ ).

MRRI-141. Shell (Mercenaria sp)

$4730 \pm 110$

MRRI-147. Shell (Mercenaria sp)

$6410 \pm 100$

MRRI-148. Shell (Mercenaria sp)

$5250 \pm 100$

MRRI-151. Shell (Mercenaria sp)

$3720 \pm 140$

MRRI-152. Shell (D robustum)

$3690 \pm 80$

MRRI-154. Shell (Mercenaria sp)

$3440 \pm 120$

MRRI-156. Shell (Mercenaria sp)

$3070 \pm 120$

MRRI-201. Shell (Mercenaria sp)

$4890 \pm 150$

MRRI-204. Shell (Anadara sp)

$3690 \pm 90$

La Costa I., Lee Co Location No. 2

Samples coll from beach ridge sand $\sim \operatorname{lm}$ above MSL $\left(26^{\circ} 41^{\prime} 06^{\prime \prime}\right.$ $\mathrm{N}, 82^{\circ} 14^{\prime} 53^{\prime \prime} \mathrm{W}$ ).

MRRI-175. Shell (Mercenaria sp)

$2650 \pm 80$

MRRI-176. Shell (Spisula sp)

$1340 \pm 110$

MRRI-180. Shell (Anadara sp)

$2260 \pm 310$

MRRI-182. Shell (Strombus sp)

$1980 \pm 120$

MRRI-183. Shell (Anadara sp)

$1830 \pm 130$

MRRI-184. Shell (Anadara sp)

$1830 \pm 110$

MRRI-188. Shell (Spisula sp)

$2550 \pm 170$

MRRI-189. Shell (Spisula sp)

$2120 \pm 150$

\section{Location No. 3}

Samples coll from beach ridge sand $\sim \operatorname{lm}$ above MSL $\left(26^{\circ} 41^{\prime} 06^{\prime \prime}\right.$ $\left.\mathrm{N}, 82^{\circ} 15^{\prime} 00^{\prime \prime} \mathrm{W}\right)$. 
MRRI-177. Shell (Mercenaria sp)

$$
\begin{array}{r}
2600 \pm 120 \\
2270 \pm 90 \\
2410 \pm 80 \\
2200 \pm 80 \\
2160 \pm 70 \\
1980 \pm 70
\end{array}
$$

MRRI-178. Shell (B carica)

MRRI-179. Shell (Anadara sp)

MRRI-185. Shell (B carica)

MRRI-186. Shell (D robustum)

MRRI-187. Shell (Mercenaria sp)

\section{Orange Cove}

Samples coll from beach ridge with poorly lithified calcarenite 0 to $50 \mathrm{~cm}$ above MLW $\left(26^{\circ} 39^{\prime} 55^{\prime \prime} \mathrm{N}, 82^{\circ} 14^{\prime} 30^{\prime \prime} \mathrm{W}\right)$.

$\begin{array}{llr}\text { MRRI-190. } & \text { Shell (Mercenaria } \text { sp) } & 2920 \pm 90 \\ \text { MRRI-191. } & \text { Shell (B carica) } & \mathbf{3 8 1 0} \pm \mathbf{2 0 0} \\ \text { MRRI-192. } & \text { Shell (Mercenaria } \text { sp) } & \mathbf{3 1 2 0} \pm \mathbf{9 0} \\ \text { MRRI-193. } & \text { Shell (Mercenaria } \text { sp) } & \mathbf{3 7 5 0} \pm \mathbf{2 3 0} \\ \text { MRRI-195. } & \text { Shell (Mercenaria } \text { sp) } & \mathbf{3 6 8 0} \pm \mathbf{1 0 0} \\ \text { MRRI-196. } & \text { Shell (Mercenaria } \text { sp) } & \mathbf{3 7 7 0} \pm \mathbf{9 0} \\ \text { MRRI-199. } & \text { Shell (Mercenaria } \text { sp) } & \mathbf{4 5 1 0} \pm \mathbf{1 1 0} \\ \text { MRRI-202. } & \text { Shell (Mercenaria } \text { sp) } & \mathbf{3 0 5 0} \pm 100 \\ \text { MRRI-203. } & \text { Shell (D robustum) } & \mathbf{3 6 4 0} \pm 130\end{array}$

\section{North Captiva I., Lee Co}

Samples coll from beach ridge sand and poorly lithified calcarenite 0 to $1 \mathrm{~m}$ above MSL $\left(26^{\circ} 35^{\prime} 50^{\prime \prime} \mathrm{N}, 82^{\circ} 13^{\prime} 10^{\prime \prime} \mathrm{W}\right)$.

MRRI-205. Shell (Mercenaria sp)

$$
\begin{array}{r}
4100 \pm 80 \\
1880 \pm 80 \\
3130 \pm 80 \\
2230 \pm 70 \\
4620 \pm 80 \\
3450 \pm 100 \\
4800 \pm 110 \\
4060 \pm 110
\end{array}
$$$$
\text { MRRI-206. Shell (Mercenaria sp) }
$$$$
\text { MRRI-207. Shell (Mercenaria sp) }
$$$$
\text { MRRI-208. Shell (Mercenaria sp) }
$$$$
\text { MRRI-209. Shell (Mercenaria sp) }
$$

MRRI-210. Shell (Mercenaria sp)

MRRI-211. Shell (Mercenaria sp)

MRRI-212. Shell (Mercenaria sp)

REFERENCES

Coleman, D D, Liu, C L, Dickerson, D R, and Frost, R R, 1972, Improvement in trimerization of acetylene to benzene for radiocarbon dating with a commerically available vanadium oxide catalyst, in Rafter, T A and Grant-Taylor, T, eds, Internatl conf on radiocarbon dating, 8th, Proc: Wellington, Royal Soc New Zealand, B50-B62.

Mathews, T D, 1976, Marine Resources Research Institute radiocarbon dates I: Radiocarbon, v 18, p 202-204.

carbon, v 20, p 436-440. 